\title{
ON A THEOREM OF A. BOREL
}

\author{
BY \\ W. H. COCKCROFT
}

1. Introduction. Let $(E, B, F, p)$ denote a fibre space in the sense of J.-P. Serre, in which the base $B$ is arcwise connected and $\pi_{1}(B)$ operates trivially on the fibre $F$. In what follows, cohomology will always mean singular (cubical) cohomology; the standard notation, $C^{*}, Z^{*}$, etc., for cochains, cocycles, etc., will be used, and coefficients will always be taken in a commutative ring $K$ with a unit element. Throughout, it will be assumed that the cohomology algebra $H^{*}(F ; K)$ is a graded tensor product of monogenic, possibly truncated, polynomial algebras with transgressive generators of positive degrees, and that $H^{*}(F ; K)$ is of finite type (i.e., has a finite (additive) $K$-basis in each dimension) with $1 \in H^{0}(F ; K)$.

My aim here is, as in [3], to study the relation between the cohomology groups of $E$ and those of $B$ and $F$. I recall that in [3] details were given of the method of Hirsch in singular cohomology theory, in which under suitable conditions, a coboundary operator $d$ is proved to exist on the tensor product $C^{*}(B ; K) \otimes_{K} H^{*}(F ; K)$, together with a map $u: C^{*}(B ; K) \otimes_{K} H^{*}(F ; K)$ $\rightarrow C^{*}(E ; K)$ which induces isomorphisms on cohomology groups. The results proved here below show that, in fact, under the conditions given above, $d$ and $\boldsymbol{u}$, in the Hirsch method, can be defined by explicit formulae in terms of cup-and cup-1-products obtained from the generators of $H^{*}(F ; K)$. These explicit formulae of course generalize the explicit formulae given in [3] up to dimension $2 n-1$ for $d$ and $u$, in case $F$ is a space of type $\nVdash(\pi, n)$.

Collected together, the results may be regarded as a "weak" generalization of a theorem of A. Borel( $\left.{ }^{1}\right)$, referred to in the title. I use the adjective "weak" because whereas Borel proves the existence of a multiplicative isomorphism in cohomology, viz. $H^{*}\left(C^{*}(B ; K) \otimes_{K} H^{*}(F ; K)\right) \approx H^{*}(E ; K)$, here I can only prove the existence of an additive isomorphism (despite the existence of a multiplicative, cup-product structure). However, I claim the results to be a generalization of the theorem of Borel, since they are valid in ordinary, singular, cohomology theory, for fibre spaces in the sense of Serre, whereas in order to obtain anti-commutative systems of cochains, and hence a multiplicative isomorphism, Borel is obliged to restrict his attention to fibre bundles, and to use Alexander-Spanier cohomology theory with compact supports. Furthermore, in the results proved here, the cohomology algebra $H^{*}(F ; K)$ of the fibre

Presented to the Society, August 26, 1958 under the title The cohomology groups of certain fibre spaces; received by the editors June 4, 1959 and, in revised form, August 5, 1960.

(1) Cf. $[1, \$ 24]$ for the statement and proof of this result. Cf. also footnote 12, p. 517, [3]. 
is no longer restricted as in Borel's result to being an exterior algebra; and finally the proofs are valid over a ring of coefficients, whereas Borel's result is valid only over the real field.

The paper is arranged as follows. First, the explicit formulae for $d$ and $u$ are given, and $u$ is proved to be an admissible map for the Hirsch technique. (The methods of [3] are used throughout, and the reader is referred to that paper for details of those methods, especially in the proof of Proposition 1.) Second, the generalization of Borel's result is completed. The paper closes with two short appendices, one on the applicability of the results when $F$ is a space of type $\mathscr{K}(\pi, n)$, and the other on the possibility of introducing a product structure into $C^{*}(B ; K) \otimes_{K} H^{*}(F ; K)$, so that the isomorphism in cohomology given by the Hirsch method becomes a multiplicative isomorphism. In this last appendix the questions involved remain open.

2. Definition of $d, u$ and the method of Hirsch. I shall denote by $x_{i}, i=1,2, \cdots$, the transgressive generators of $H^{*}(F ; K)$. Since $H^{*}(F ; K)$ is the tensor product of monogenic polynomial algebras with the $x_{i}$ as their respective generators, a typical additive $K$-basis element of $H^{*}(F ; K)$ is $x=x_{i_{1}}^{\tau_{1}} x_{i_{2}}^{\tau_{2}} \cdots x_{i_{n}}^{\tau_{n}}, r_{i}>0, i_{1}<i_{2}<\cdots<i_{n}$. Let $y_{i}$ denote a chosen cocycle in the transgression of $x_{i}$, with $c_{i}$ the transgression cochain, so that if $\partial_{E}$ is the coboundary operator in $E, \partial_{E} c_{i}=p^{*} y_{i}$, where $p^{*}=C^{*}(B ; K) \rightarrow C^{*}(E ; K)$ is the homomorphism induced by $p: E \rightarrow B$.

The definitions and proofs which follow will all be inductive in character. I state once for all that the inductive assumptions will be valid on additive basis elements of $H^{*}(F ; K), x=x_{i_{1}}^{r_{1}} x_{i_{2}}^{\tau_{2}} \cdots x_{i_{n}}^{\tau_{n}}, i_{1}<i_{2}<\cdots<i_{n}$, as above, with $n \leqq l, r_{n} \leqq k$, for some integers $l, k$, and that the inductive extension will be to basis elements $x x_{i}$ with $i \geqq i_{n}$.

Greek letters will always denote the degrees of elements with corresponding Roman letters; thus, without explicit statement of these facts, it will be assumed that $\beta$ denotes the degree of $b, \beta^{\prime}$ that of $b^{\prime}, \xi$ that of $x, \xi_{i}$ that of $x_{i}$, etc. Notice that, in consequence, certain relations will hold among these Greek symbols; e.g., since by definition $y_{i}$ is a cocycle in the transgression of $x_{i}$, degree $\left(y_{i}\right)=$ degree $\left(x_{i}\right)+1$, i.e., $\eta_{i}=\xi_{i}+1$. To help the printer I denote by $\wp(n)$ the parity $(-1)^{n}$ of an integer $n$.

2.1. Definition of $d$. In order to define the differential operator $d$ in $L=C^{*}(B ; K) \otimes_{K} H^{*}(F ; K)$, I shall require, in addition to the usual (cup) product in $L$, viz.

$$
(b \otimes x)\left(b^{\prime} \otimes x^{\prime}\right)=\varnothing\left(\xi \beta^{\prime}\right)\left(b b^{\prime} \otimes x x^{\prime}\right),
$$

a product which is, to within a \pm sign, the cup-1-product in $B$ with coeffcients in $H^{*}(F ; K)$. (The latter being paired to itself in the natural way.) This new product will be denoted by $\mathrm{O}$. It is defined by setting

$$
(b \otimes x) \circ\left(b^{\prime} \otimes x^{\prime}\right)=\varnothing\left(\xi\left(\beta^{\prime}-1\right)\right)\left\{\left(b \cup_{1} b^{\prime}\right) \otimes x x^{\prime}\right\},
$$


and extending by linearity; (here $\cup_{1}$ denotes the cup-1-product in $B$, and of course $\left.x \in H^{\xi}(F ; K), b^{\prime} \in C^{\beta^{\prime}}(B ; K)\right)$.

Denote by $\partial_{B}$ the coboundary operator in $B$. An additive homomorphism $d: L \rightarrow L$ is defined by setting, firstly,

$$
d(b \otimes 1)=\partial_{B} b \otimes 1,
$$

and, inductively, for the additive $K$-basis elements of $H^{*}(F ; K)$,

$$
\begin{aligned}
& d\left(1 \otimes x_{i}\right)=y_{i} \otimes 1, \\
& d\left(1 \otimes x x_{i}\right)=\{d(1 \otimes x)\}\left(1 \otimes x_{i}\right)+\wp\left(\xi \xi_{i}\right)\left(y_{i} \otimes x\right) \\
&+\wp\left(\xi_{i}+1\right)\{d(1 \otimes x)\} \circ\left\{y_{i} \otimes 1\right\} .
\end{aligned}
$$

The definition is completed in the usual way, viz. by linearity, and by setting

$$
d(b \otimes x)=\partial_{B} b \otimes x+8(\beta)\{b \otimes 1\}\{d(1 \otimes x)\},
$$

for all $b \in C^{*}(B ; K)$ and all additive $K$-basis elements $x \in H^{*}(F ; K)$.

2.2. Definition of $u: L \rightarrow C^{*}(E ; K)$. Following the procedure of the method of Hirsch $[3, \S 2]$, the homomorphism

$$
\mathbf{u}: C^{*}(B ; K) \otimes_{\mathrm{K}} H^{*}(F ; K) \rightarrow C^{*}(E ; K)
$$

is defined by first defining a homomorphism

$$
u: C^{0}(B ; K) \otimes_{K} H^{*}(F ; K) \rightarrow C^{*}(E ; K),
$$

and then, by setting

$$
u(b \otimes x)=\left(p^{*} b\right)\{u(1 \otimes x)\}
$$

The homomorphism $u$ will be defined inductively on the additive $K$-basis elements of $H^{*}(F ; K)$, and extended by linearity, by setting

$$
\begin{aligned}
u\left(1 \otimes x_{i}\right) & =c_{i} \\
u\left(1 \otimes x x_{i}\right) & =\{u(1 \otimes x)\} c_{i}+\varphi\left(\xi_{i}+1\right)\left\{u(1 \otimes x) \cup_{1} \partial_{E} c_{i}\right\},
\end{aligned}
$$

where $\cup_{1}$ here denotes the cup-1-product in $E$, and, as before, $\partial_{E}$ is the coboundary operator in $E$ and $c_{i}$ is the transgression cochain associated with $x_{i}$, i.e., $p^{*} y_{i}=\partial_{E} c_{i}$. It is obvious that $u$, so defined, preserves degrees.

LEMMA 1. $u d=\partial_{E} u$.

Certainly $u d\left(1 \otimes x_{i}\right)=\partial_{E} u\left(1 \otimes x_{i}\right)$, for both are equal $p^{*} y_{i}$. Assume inductively, therefore, that $u d(1 \otimes x)=\partial_{E} u(1 \otimes x)$. We require to consider the truth of the lemma for elements $1 \otimes x x_{i}$; the proof of the lemma will then follow from linearity considerations. 
By definition $d(1 \otimes x)$ is a finite sum of elements of the form $\left(b \otimes x^{\prime}\right) \in L$, where $b \in C^{\beta}(B ; K), x^{\prime} \in H^{\xi^{\prime}}(F ; K)$, and $\beta+\xi^{\prime}=\xi+1$. Let $\sum\left(b \otimes x^{\prime}\right)$ denote this finite sum; and indeed throughout the proof of the lemma let $\sum$ always denote summation with respect to the pairs $b, x^{\prime}$ appearing in $d(1 \otimes x)$. By definition

$$
u\left(1 \otimes x x_{i}\right)=\{u(1 \otimes x)\} c_{i}+\varnothing\left(\xi_{i}+1\right)\left\{u(1 \otimes x) \cup_{1} \partial_{E} c_{i}\right\}
$$

Thus

$$
\begin{aligned}
& \partial_{E} u\left(1 \otimes x x_{i}\right) \\
& =\left\{\partial_{E} u(1 \otimes x)\right\} c_{i}+\varphi(\xi)\{u(1 \otimes x)\}\left(\partial_{E} c_{i}\right)+\varphi\left(\xi_{i}+1\right)\left[\varphi\left(\xi+\xi_{i}\right)\{u(1 \otimes x)\}\left(\partial_{E} c_{i}\right)\right. \\
& \left.+\wp\left(\xi\left(\xi_{i}+1\right)+\xi+\xi_{i}+1\right)\left(\partial_{E} c_{i}\right)\{u(1 \otimes x)\}+\left\{\partial_{E} u(1 \otimes x)\right\} \cup_{1} \partial_{E} c_{i}\right] \\
& =\left\{\partial_{E} u(1 \otimes x)\right\} c_{i}+\varnothing\left(\xi \xi_{i}\right)\left(\partial_{E} c_{i}\right)\{u(1 \otimes x)\}+\varnothing\left(\xi_{i}+1\right)\left\{\partial_{E} u(1 \otimes x)\right\} \cup_{1} \partial_{E} c_{i} \\
& =\{u d(1 \otimes x)\} c_{i}+8\left(\xi \xi_{i}\right)\left(p^{*} y_{i}\right)\{u(1 \otimes x)\}+\wp\left(\xi_{i}+1\right)\{u d(1 \otimes x)\} \cup_{1} p^{*} y_{i} \\
& =u\left(\sum\left(b \otimes x^{\prime}\right)\right) c_{i}+\varphi\left(\xi \xi_{i}\right) u\left(y_{i} \otimes x\right)+\varphi\left(\xi_{i}+1\right)\left\{u\left(\sum\left(b \otimes x^{\prime}\right)\right)\right\} \cup_{1} p^{*} y_{i} \\
& =\sum\left(p^{*} b\right)\left\{u\left(1 \otimes x^{\prime}\right)\right\} c_{i}+\varphi\left(\xi \xi_{i}\right) u\left(y_{i} \otimes x\right)+\varphi\left(\xi_{i}+1\right) \sum\left\{\left(p^{*} b\right) u\left(1 \otimes x^{\prime}\right)\right\} \cup_{1} p^{*} y_{i} \\
& =\sum p^{*} b\left[u\left(1 \otimes x^{\prime} x_{i}\right)+\varphi\left(\xi_{i}\right)\left\{u\left(1 \otimes x^{\prime}\right)\right\} \cup_{1} p^{*} y_{i}\right] \\
& +\wp\left(\xi \xi_{i}\right) u\left(y_{i} \otimes x\right)+\sum \varnothing\left(\xi_{i}+1\right)\left\{\left(p^{*} b\right) u\left(1 \otimes x^{\prime}\right)\right\} \cup_{1} p^{*} y_{i} .
\end{aligned}
$$

Using the Hirsch identity [5] for cup-1-products, this expression

$$
\begin{aligned}
& =u\left[\sum\left(b \otimes x^{\prime} x_{i}\right)+\wp\left(\xi \xi_{i}\right)\left(y_{i} \otimes x\right)\right]+\sum \wp\left(\xi_{i}+1+\xi^{\prime} \xi_{i}\right)\left\{p^{*} b \cup_{1} p^{*} y_{i}\right\} u\left(1 \otimes x^{\prime}\right) \\
& =u\left[\sum\left(b \otimes x^{\prime} x_{i}\right)+\wp\left(\xi \xi_{i}\right)\left(y_{i} \otimes x\right)+\sum \wp\left(\xi_{i}+1+\xi^{\prime} \xi_{i}\right)\left(b \cup_{1} y_{i}\right) \otimes x^{\prime}\right] .
\end{aligned}
$$

But, on the other hand

$u d\left(1 \otimes x x_{i}\right)$

$$
\begin{aligned}
&=u\left[\left\{\sum\left(b \otimes x^{\prime}\right)\right\}\left(1 \otimes x_{i}\right)+\right. \wp\left(\xi \xi_{i}\right)\left(y_{i} \otimes x\right) \\
&\left.+\wp\left(\xi_{i}+1\right) \sum \wp\left(\xi^{\prime}\left(\eta_{i}-1\right)\right)\left(b \cup_{1} y_{i}\right) \otimes x^{\prime}\right] \\
&=u\left[\sum\left(b \otimes x^{\prime} x_{i}\right)+\wp\left(\xi \xi_{i}\right)\left(y_{i} \otimes x\right)+\sum \wp\left(\xi_{i}+1+\xi^{\prime} \xi_{i}\right)\left(b \cup_{1} y_{i}\right) \otimes x^{\prime}\right] .
\end{aligned}
$$

Thus, as required $\partial_{E} u\left(1 \otimes x x_{i}\right)=u d\left(1 \otimes x x_{i}\right)$.

2.3. The admissibility of $u$ in the Hirsch method. It is now a relatively simple matter to prove the

Proposition 1. The homomorphism $u: C^{0}(B ; K) \otimes_{K} H^{*}(F ; K) \rightarrow C^{*}(E ; K)$ is an admissible map for the Hirsch method.

We require to show that $u$, defined as above, satisfies condition (i) of the 
assertion of the Hirsch method, given in [3, p. 511]. Lemma 2 above implies immediately that the second part of the condition is satisfied. Using the notation of [3] it therefore remains only to show that $\mu \beta u=\phi^{* *}$. (I shall not repeat the definitions implicit in this use of the notation of [3]. As stated in the Introduction above, the reader is referred to [3] for such details as are necessary.)

Again the proof which follows will be inductive in character. Still using the notation of $[3]$, note that, in the first place

$$
\begin{aligned}
\lambda i^{*} u\left(1 \otimes x_{i}\right) & =\lambda i^{*} c_{i} \\
& =x_{i}
\end{aligned}
$$

for any multiplicative generator of $H^{*}(F ; K)$. Consider $\lambda i^{*} u\left(1 \otimes x x_{i}\right)$, assuming by induction that $\lambda i^{*} u(1 \otimes x)=1 \otimes x$. We have

$$
\begin{aligned}
\lambda i^{*} u\left(1 \otimes x x_{i}\right) & =\lambda i^{*}\left[\{u(1 \otimes x)\} c_{i}+\wp\left(\xi_{i}+1\right)\{u(1 \otimes x)\} \cup_{1} \partial_{E} c_{i}\right] \\
& =\left\{\lambda i^{*} u(1 \otimes x)\right\}\left\{\lambda i^{*} c_{i}\right\}+\wp\left(\xi_{i}+1\right)\left\{\lambda i^{*} u(1 \otimes x)\right\} \cup_{1}\left\{\lambda i^{*} p^{*} y_{i}\right\} \\
& =\left\{\lambda i^{*} u(1 \otimes x)\right\}\left\{\lambda i^{*} c_{i}\right\},
\end{aligned}
$$

since $i^{*} p^{*} y_{i}=0$; whence, as required,

$$
\lambda i^{*} u\left(1 \otimes x x_{i}\right)=(1 \otimes x)\left(1 \otimes x_{i}\right)=1 \otimes x x_{i} .
$$

Thus, for any additive $K$-basis element $x \in H^{*}(F ; K)$, it follows from the remarks in $[3, S 2]$ that,

$$
1 \otimes x=\lambda i^{*} u(1 \otimes x)=\lambda \psi^{*} \beta u(1 \otimes x)=\psi^{* *} \mu \beta u(1 \otimes x),
$$

i.e.,

$$
\phi^{* *}(1 \otimes x)=\phi^{* *} \psi^{* *} \mu \beta u(1 \otimes x)=\mu \beta u(1 \otimes x),
$$

as required.

Now it follows immediately from the remarks in $[3, S 2]$ that $\mu \beta u(b \otimes 1)$ $=\mu \beta p^{*} b=\phi^{* *}(b \otimes 1)$ for all $b \in C^{*}(B, K)$. Thus from $[3, \mathrm{~S} 4]$,

$$
\begin{aligned}
\mu \beta u(b \otimes x) & =\mu \beta\left(\left\{p^{*} b\right\}\{u(1 \otimes x)\}\right) \\
& =\left\{\mu \beta p^{*} b\right\}\{\mu \beta u(1 \otimes x)\} \\
& =\left\{\phi^{* *}(b \otimes 1)\right\}\left\{\phi^{* *}(1 \otimes x)\right\} \\
& =\phi^{* *}\{(b \otimes 1)(1 \otimes x)\} \\
& =\phi^{* *}(b \otimes x),
\end{aligned}
$$

and the required result follows from linearity considerations. 
It follows immediately then, from the method of Hirsch, and Lemma 1 above, that $u$ is a monomorphism, and that the coboundary operator $d$ defined above is the (unique) Hirsch coboundary operator. Thus $\mathbf{u}$ induces an additive isomorphism between the graded cohomology $K$-module of $C^{*}(B ; K) \otimes_{K} H^{*}(F ; K)$, computed by using the coboundary operator $d$, and the graded cohomology $K$-module $H^{*}(E ; K)$. This completes the generalization of the first part of the result of A. Borel.

To generalize the second part of the Borel result, I require to prove the existence of homomorphisms $q$ and $r$ such that the following diagram is commutative:

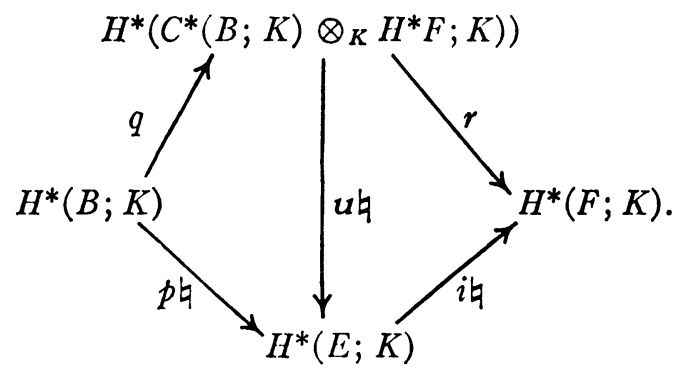

The homomorphisms $p^{\natural}$ and $i^{\natural}$ in the diagram are of course induced by $p: E \rightarrow B$ and the injection $i: F \rightarrow E$, just as $\mathbf{u}^{\natural}$ is induced by $u$.

A trivial argument concerning the degrees of the terms involved shows that an element of $1 \otimes H^{*}(F ; K)$ can never be a coboundary in $L$ $=C^{*}(B ; K) \otimes_{K} H^{*}(F ; K)$. Thus a cohomology class of $L$ contains at most one cocycle of $1 \otimes H^{*}(F ; K)$. Denote therefore by $r$ the isomorphism of $L$ into $H^{*}(F ; K)$ defined by associating this cocycle with any such cohomology class. Further, let $q: H^{*}(B ; K) \rightarrow H^{*}(L)$ denote the homomorphism induced by the mapping $b \rightarrow b \otimes 1$, for all $b \in C^{*}(B ; K)$. With these definitions the homomorphisms in the diagram correspond precisely to those in the Borel result.

To prove that the diagram is commutative, let us first for the sake of notation denote by $z$ the cohomology class of any cocycle $z$. Thus for example if $1 \otimes x^{\prime} \in 1 \otimes H^{*}(F ; K)$ is a cocycle in $L$, then $r\left(1 \otimes x^{\prime}\right)=x^{\prime}$. Then the Proposition 1 above implies that $u^{\natural} q b=u(b \otimes 1)=p^{*} b=p^{\natural} b$. Also, again using the notation of [3] as in the proof of Proposition 1 above,

$$
\begin{aligned}
i_{\natural} u^{\natural}\left(1 \otimes x^{\prime}\right) & =i \natural\left\{u\left(1 \otimes x^{\prime}\right)\right\}=i^{*} u\left(1 \otimes x^{\prime}\right) \\
& =\lambda i^{*} u\left(1 \otimes x^{\prime}\right)=1 \otimes x^{\prime}
\end{aligned}
$$

which, identifying $1 \otimes H^{*}(F ; K)$ with $H^{*}(F ; K)$ is equal to $r\left(1 \otimes \mathbf{x}^{\prime}\right)$. Thus the diagram is commutative as required.

Appendix 1. Applicability of results. The cohomology algebra of a space of type $\mathfrak{K}(\pi, n)$ over the field $Z_{p}$ of integers $\bmod p$ is, by the results of $\mathrm{H}$. Cartan 
[2], a tensor product of an exterior algebra (over a vector space with elements of odd degrees) with a symmetric algebra (over a vector space with elements of even degrees). Thus certainly fibre spaces with fibre of type $\mathfrak{K}(\pi, n)$ will yield examples in which the result proved above is applicable, provided the multiplicative generators in the cohomology algebra $H\left(\pi, n ; Z_{p}\right)$ are transgressive. That this is indeed so follows from results of $\mathrm{H}$. Cartan loc. cit., Exposé 14. Thus, by definition, these generators are dual to the generators in the homology algebra $H_{*}\left(\pi, n ; Z_{p}\right)$ and are therefore orthogonal to the decomposable elements in this homology algebra. In consequence, then, of the last remark in [2, Exposé 14, p. 12], the generators of $H^{*}\left(\pi, n ; Z_{p}\right)$ are in the image of the suspension homomorphism: $H^{q+1}\left(\pi, n+1 ; Z_{p}\right)$ $\rightarrow H^{q}\left(\pi, n ; Z_{p}\right)$, i.e., they are transgressive in the fibre space of paths on a space of type $\mathcal{K}(\pi, n+1)$. As noted in the lemma of [3, §3], the generators are therefore "universally" transgressive, i.e., transgressive in any fibre space with fibre of type $\varkappa(\pi, n)$.

An alternative, possibly more direct, proof of this result can, of course, be obtained by means of the Cartan theory of constructions. One requires the generators of $H^{*}\left(\pi, n ; Z_{p}\right)$ to be transgressive in the $W$-bar construction $\left(^{2}\right)$ $(K(\pi, n), L(\pi, n+1), K(\pi, n+1))$. This construction is equivalent, when normalised, to the (ordinary) bar construction, and this in turn has the homology and cohomology of the tensor product of "basic" acyclic constructions of Cartan $\left(^{(3)}\right.$. In these basic constructions it is easy to verify that the multiplicative generators in cohomology are transgressive and of course the equivalence of the constructions together with the "projections" onto the basic constructions enable one to carry back to the original $W$-bar construction the fact that the generators are transgressive.

Appendix 2. Two open questions. It appears difficult to decide whether or not $\mathfrak{u}^{\natural}: H^{*}(L) \approx H^{*}(E ; K)$ can be made multiplicative. I am, for instance, unable to answer the following two questions.

(i) Is there a product structure induced in $H^{*}(L)$ by the product

of $L$ ?

$$
(b \otimes x)\left(b^{\prime} \otimes x^{\prime}\right)=\varnothing\left(\xi \beta^{\prime}\right)\left(b b^{\prime} \otimes x x^{\prime}\right)
$$

This seems unlikely but I have no counter-example.

(ii) Even if (i) is false, is $u L \subset C^{*}(E ; K)$ closed with respect to the cupproduct multiplication in $C^{*}(E ; K)$, so that a multiplication can be imposed on $L$ making $u$, and hence $u^{\natural}$, multiplicative?

\section{BIBLIOGRAPHY}

1. A. Borel, Sur la cohomologie des espaces fibrés principaux et des espaces homogènes de groupes de Lie compacts, Ann. of Math. vol. 5 (1953) pp. 115-207.

(2) Cf. H. Cartan [2, Exposé 13].

(3) I.e. constructions on, respectively, monogenic exterior algebras, and polynomial algebras, over $Z_{p}$; cf. H. Cartan, loc. cit., Exposé 9. 
2. H. Cartan, Seminaire H. Cartan de l'Ecole Normale Superieure, (mimeographed) 19541955, Paris.

3. W. H. Cockcroft, The cohomology groups of a fibre space with fibre a space of type $\mathscr{T K}(\pi, n)$. II, Trans. Amer. Math. Soc. vol. 91 (1959) pp. 505-524.

4. G. Hirsch, Sur les groupes d'homologie des espaces fibres, Bull. Soc. Math. Belgique vol. 6 (1953) pp. 79-96.

5. - Quelques proprittes des produits de Steenrod, C. R. Acad. Sci. Paris vol. 241 (1955) pp. 923-925.

6. J.-P. Serre, Homologie singulière des espaces fibrts. Applications, Ann. of Math. vol. 54 (1951) pp. 425-505.

UNIVERSITY OF SOUTHAMPTON,

SOUTHAMPTON, ENGLAND 